\title{
Effects of $\mathrm{H3}$ and $\mathrm{H} 4$ Histones Acetylation and Bindings of CREB Binding Protein and p300 at the Promoter on Hepatic Expression of $\gamma$-glutamyltransferase Gene in a Streptozotocin-Induced Moderate Hypoinsulinemic Rat Model
}

\author{
Tomoyuki TANAKA ${ }^{1}$, Takahiro MIZUNO ${ }^{2}$, Tomoyuki NAKAGAWA ${ }^{1,2}$, Takashi \\ HAYAKAWA ${ }^{1,2}$, Masaya SHIMADA ${ }^{1,2}$
}

${ }^{1}$ Division of Life Science for Food, Department of Life Science and Chemistry, Graduate School of Natural Science and Technology, Gifu University, Gifu, Japan, ${ }^{2}$ Department of Applied Life Science, Faculty of Applied Biological Sciences, Gifu University, Gifu, Japan

Received November 11, 2020

Accepted March 23, 2021

Epub Ahead of Print May 12, 2021

\begin{abstract}
Summary
Gamma-glutamyltransferase (GGT), a marker of liver disease, has been shown to be associated with increased risk of diabetes and relative insulin secretion deficiency. However, the mechanism of hepatic Ggt regulation has not been explored fully. In this study, we made a concerted effort to understand the mechanism by investigating the effects of acetylation of histones $\mathrm{H} 3$ and $\mathrm{H} 4$, and bindings of histone acetyltransferases, CREB binding protein (CBP) and p300, at the Ggt promoter on the regulation of the expression of Ggt gene in the livers of streptozotocin (STZ)-induced moderate hypoinsulinemia rat model. The rats treated with STZ showed remarkably higher serum GGT level and hepatic Ggt/GGT expression than the untreated control rats. Furthermore, the acetylation of histones $\mathrm{H} 3$ and $\mathrm{H} 4$, and the binding of CBP not p300 at the Ggt promoter regions were significantly higher in the livers of STZ rats than those of the control rats. These results suggest that an enhanced hepatic expression of $\mathrm{Ggt}$ is associated with increased acetylation of histones $\mathrm{H} 3$ and $\mathrm{H} 4$ and $\mathrm{CBP}$ binding at the Ggt promoter in STZ-induced moderate hypoinsulinemic rats.
\end{abstract}

\section{Key words}

Acetylated histone $\bullet$ CBP • GGT • Liver • Streptozotocin

\section{Corresponding author}

M. Shimada, Department of Applied Life Science, Faculty of Applied Biological Sciences, Gifu University, 1-1 Yanagido, Gifu-shi, Gifu 501-1193, Japan. E-mail: mshimada@gifu-u.ac.jp
Gamma-glutamyltransferase (GGT) plays a key role in the extracellular catabolism of the major antioxidant, glutathione. Its increased circulating level by oxidative stress has been reported as a marker for hepatic injuries, such as liver dysfunction induced by excessive alcohol intake (Kunutsor 2016). Furthermore, human observational studies via a systematic review have reported that the circulating levels of GGT are associated with an increased risk of type 2 diabetes (Kunutsor et al. 2014). It has also been shown that the increased levels of GGT in the blood are related to the attenuated function of pancreatic $\beta$-cells in young obese subjects (Wang et al. 2013). However, in adult patients with type 1 diabetes, Arkkila et al. (2001) could not establish its association with diabetes duration, body mass index, and hemoglobin Alc instead showed its association with retinopathy and neuropathy. Collectively, these studies suggest that the elevated circulating levels of GGT could be associated with relative but not absolute insulin secretion deficiency. Furthermore, an in vitro study has shown that insulin treatment decreases the expression of the $G G T$ gene in human hepatocytes, HepG2 (Honma et al. 2017), suggesting that increased insulin secretion downregulates the expression of the $G G T$ gene in the liver. However, the regulation of the expression of $G G T$ is currently poorly understood. Therefore, we speculate that a better understanding of the regulatory mechanisms of hepatic 
GGT expression under relatively deficient insulin secretion could help develop the key strategies for the management of diabetes.

Gene expression is regulated by histone modifications, such as acetylation and methylation, and transcription factors (Schübeler et al. 2004). In particular, enhanced gene expression is closely related to the hyperacetylation of histones $\mathrm{H} 3$ and $\mathrm{H} 4$ in the euchromatin region of the genome (Roh et al. 2005). In vivo studies using type 2 diabetic mice $(\mathrm{db} / \mathrm{db})$ models have shown an increase in the hepatic expression of gluconeogenic genes, such as phosphoenolpyruvate carboxykinase 1 (Pckl), and acetylation of histone $\mathrm{H} 3$ on the gluconeogenic genes (Ravnskjaer et al. 2013). In addition, Suzuki et al. (2015) have reported that hepatic expression of fatty acid synthase gene and acetylation of histones $\mathrm{H} 3$ and $\mathrm{H} 4$ on the fatty acid synthase gene are increased in SHR/NDmc-cp rats, a spontaneously hypertensive, obese and diabetic model of rats. However, the effects of histone acetylation on the expression of the Ggt gene in the liver of hypoinsulinemic models have not yet been reported.

In the present study, we examined the effects of acetylation of histones $\mathrm{H} 3$ and $\mathrm{H} 4$ on the Ggt promoter in streptozotocin (STZ)-induced moderate (not severe) hypoinsulinemia rat model on the expression of the Ggt gene. Furthermore, it is known that CREB binding protein (CBP) and p300, the histone acetyltransferases bind to acetylated histones and nuclear transcription factors to mediate the recruitment of the transcriptional complex (Chan and La Thangue 2001). Therefore, to elucidate their role in regulating the expression of the $G G T$ gene, we investigated whether the bindings of CBP and p300 to the Ggt promoter are altered in the liver of the hypoinsulinemic rats.

Six-week-old male Wistar/ST rats were purchased from Japan SLC (Shizuoka, Japan) and divided into control and STZ groups. Control group rats $(n=6)$ were intraperitoneally administered a single dose of saline. To induce moderate hypoinsulinemia, STZ group rats $(n=7)$ were intraperitoneally administered a single low dose of STZ (40 mg/kg body weight; FUJIFILM Wako Pure Chemical Corporation, Osaka, Japan) dissolved in saline, prepared immediately (within $5 \mathrm{~min}$ ) before the administration. At 5 days after treatment, we confirmed that the random serum glucose level in the STZ group rats was within the range of $300-500 \mathrm{mg} / \mathrm{dl}$. The rats were given free access to a laboratory chow diet (MF; Oriental Yeast, Tokyo, Japan) and water throughout the acclimation and experimental periods. At 30 days after treatment, the rats were fasted for $4 \mathrm{~h}$ and euthanized via cardiac puncture under isoflurane inhalation anesthesia, and blood and liver tissue were collected for subsequent assays. All animal care and experimental procedures were approved by the Gifu University Animal Care and Usage Committee.

Concentrations of serum biochemical parameters (glucose, triglyceride, insulin, glucagon, glutamic pyruvic transaminase (GPT), and GGT) were measured using commercial kits (Glucose CII Test Wako kit, Triglyceride E-test Wako kit, LBIS Insulin-Rat ELISA kit (U-E type), Transaminase CII Test kit; all from FUJIFILM Wako Pure Chemical Corporation, Glucagon enzyme immunoassay (EIA) Kit; Yanaihara Institute, Shizuoka, Japan, and GGT Activity Colorimetric Assay Kit; Bio Vision, CA, USA). Hepatic triglycerides were determined following the method described by Shimada et al. (2019).

Hepatic total RNA was extracted and converted to cDNA using commercial kits. Real-time PCR was conducted as described by Shimada et al. (2019). The primers used were as follows:

\section{Pck1 (5'-GATGACATTGCCTGGATGAA-3',} 5'-AACCGTTTTCTGGGTTGATG-3'),

Gpt (5'-CAGGAGGGCACCTATCATTT-3', 5'-TTGGCATGGAAGTGACTGAG-3'),

Ggt (5'-ACAGCCCAGATTGTGAAAGAC-3', 5'-TCCGCACGATAGTTGTTAAGG-3'), and

$36 b 4$ (5'-CGAGAAGACCTCTTTCTTCCAA-3', 5'-AGTCTTTATCAGCTGCACATCG-3').

Relative mRNA levels were normalized to the housekeeping gene $36 b 4$ using the $2^{-\Delta \Delta \mathrm{CT}}$ method.

Preparation of hepatic protein lysates and subsequent immunoblotting were conducted following Ichigo et al. (2019). The primary antibodies used were anti-GGT antibody (Gene Tex, CA, USA) and antiTATA-binding protein (TBP; GeneTex). TBP was used as a loading control.

Hepatic chromatin and subsequent chromatin immunoprecipitation (ChIP) were prepared following the method described by Shimada et al. (2019). The antibodies used were as follows: anti-acetyl-histone H3 (Millipore, CA, USA), anti-acetyl-histone H4 (Millipore), CBP (Santa Cruz Biotechnology, TX, USA), p300 (Santa Cruz Biotechnology), or normal rabbit/mouse $\mathrm{IgG}$ (FUJIFILM Wako Pure Chemical Corporation). Immunoprecipitated DNA and input DNA were subjected to real-time PCR. The primers, which amplified three $G g t$ promoter regions, were as follows:

Ggt-900 (5'-CCTTTGAAGGGTTTTCCAGTG-3', 5'-TCCTGGTGATGTCCACAGTTT-3'), 
Ggt-700 (5'-CTTGTTGACCTTGGGCATCT-3', 5'-GGACAGTTCCTTTGCCTCTTT-3'), and

Ggt-350 (5'-TGGAGATTCCAGACAGCATAGA-3', 5'-TCACACAGATCTGAAGCCACTT-3').

ChIP signals were normalized to the corresponding input signals using the $2^{-\Delta \Delta C T}$ method.

Values are expressed as mean \pm SEM. Differences between the two groups were evaluated using the Student's $t$-test. $P<0.05$ indicated statistical significance.

The body weight gain, levels of hepatic triglycerides and serum insulin were significantly lower, whereas the levels of serum glucose, triglycerides, glucagon, GPT - another marker for hepatic damage, and GGT were significantly higher in STZ rats than in control rats (Table 1).

Hepatic expression levels of Pckl, Gpt, and Ggt genes were significantly higher in STZ rats than in the control rats (Fig. 1A). Similarly, the hepatic expression level of GGT was significantly higher in STZ rats than in the control rats (Fig. 1B).

Table 1. Physiological and biochemical parameters in control and STZ-induced moderate hypoinsulinemic rats.

\begin{tabular}{lcc}
\hline & Control & STZ \\
\hline $\begin{array}{l}\text { Body weight gain }(g) \\
\text { Liver }\end{array}$ & $132 \pm 6$ & $82 \pm 7^{* * *}$ \\
$\quad$ & \\
Weight (g) & $12.6 \pm 0.4$ & $12.1 \pm 0.4$ \\
Triglycerides (mg/g liver) & $53.6 \pm 4.3$ & $35.9 \pm 2.1^{* *}$ \\
Serum & & \\
Glucose (mg/dl) & $141 \pm 6$ & $349 \pm 31^{* * *}$ \\
Triglycerides (mg/dl) & $48.7 \pm 3.6$ & $74.8 \pm 10.0^{*}$ \\
Insulin (pg/ml) & $564 \pm 154$ & $66 \pm 26^{* *}$ \\
Glucagon (pg/ml) & $369 \pm 35$ & $552 \pm 41^{* *}$ \\
GPT (U/l) & $11.1 \pm 0.5$ & $18.1 \pm 1.5^{* *}$ \\
GGT (U/l) & $1.25 \pm 0.02$ & $1.86 \pm 0.13^{* *}$ \\
\hline
\end{tabular}

Values are expressed as means \pm standard error of the mean $(\mathrm{n}=6-7)$. $* * * P<0.001 ; * * P<0.01 ; * P<0.05$, significantly different from the control group (Student's $t$-test). Control, control rats treated with vehicle; STZ, moderate hypoinsulinemic rats treated with streptozotocin; GPT, glutamic pyruvic transaminase; GGT, y-glutamyltransferase.

Moreover, hepatic acetylation of histone $\mathrm{H} 3$ on the $-900 \mathrm{bp}$ promoter region, acetylation of histone $\mathrm{H} 4$ on the $-900 \mathrm{bp},-700 \mathrm{bp}$, and $-350 \mathrm{bp}$ promoter regions, and binding of CBP to the $-900 \mathrm{bp}$ and $-700 \mathrm{bp}$ promoter regions in the Ggt gene were significantly higher in the STZ rats than the control rats (Fig. 1C, 1D, and 1E), whereas binding of $\mathrm{p} 300$ to the promoter regions did not differ significantly between the two groups (Fig. 1F).

In this study, we observed higher hepatic expression of $\mathrm{Ggt} / \mathrm{GGT}$ and serum level of GGT in the STZ rats than in control rats, which suggested that though GGT is expressed in many tissues, the increased GGT level in the blood could be partially attributable to enhanced hepatic expression of Ggt/GGT.

For the first time, this study revealed that the binding of $\mathrm{CBP}$ and the acetylation of histones $\mathrm{H} 3$ and $\mathrm{H} 4$ were increased in the promoter regions of Ggt in the liver of the STZ-induced moderate hypoinsulinemic rats; however, it remains unclear which signals contribute to the enhanced hepatic expression of the Ggt gene. Shoukry (1988) showed that the treatment with insulin reduces the GGT levels in the blood of STZ-induced type 1 diabetic rats. In addition, Honma et al. (2017) reported that insulin treatment decreases the expression of Ggt and Gpt genes and gluconeogenic enzyme genes, such as $P c k l$, and acetylation of histones $\mathrm{H} 3$ and $\mathrm{H} 4$ on the Gpt and Pckl genes in HepG2 cells. Moreover, He et al. (2009) demonstrated that insulin treatment represses Pckl expression via phosphorylation of $\mathrm{CBP}$ at serine 436, which inactivates CBP in mouse liver. Collating the findings of this study with those of previously reported studies, it can be inferred that moderate hypoinsulinemia could be associated with dephosphorylation of CBP at serine 436 and recruitment of CBP to increase acetylation of histones $\mathrm{H} 3$ and $\mathrm{H} 4$ in the promoter region of Ggt. However, this should be validated through further studies by investigating the effects of insulin administration to STZ-induced moderate hypoinsulinemic rats. 
(A)

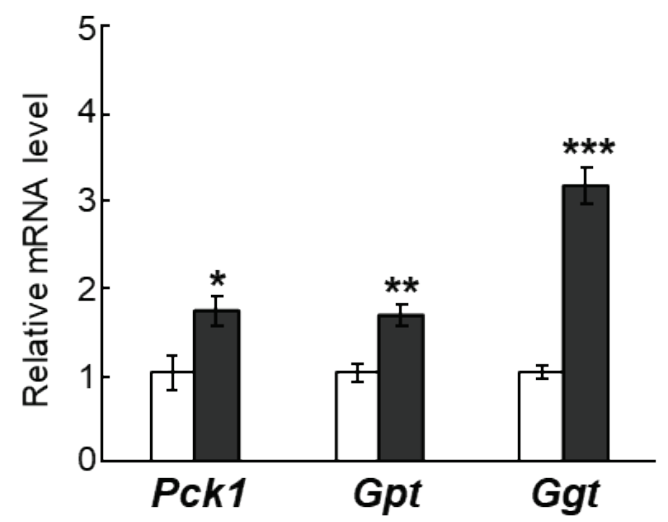

(C)



(E)

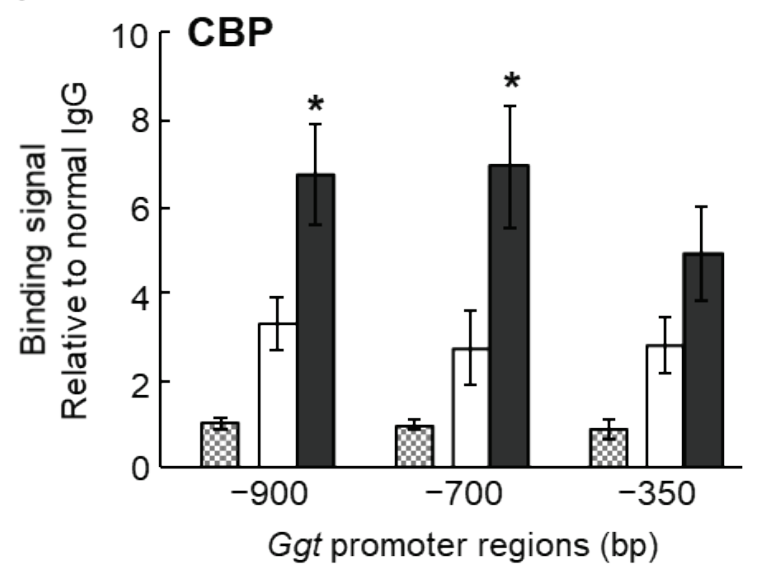

(B)
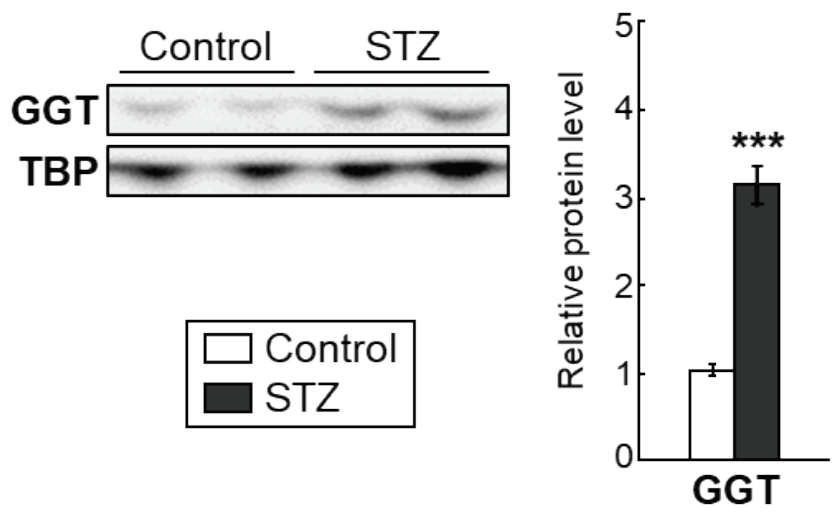

(D)

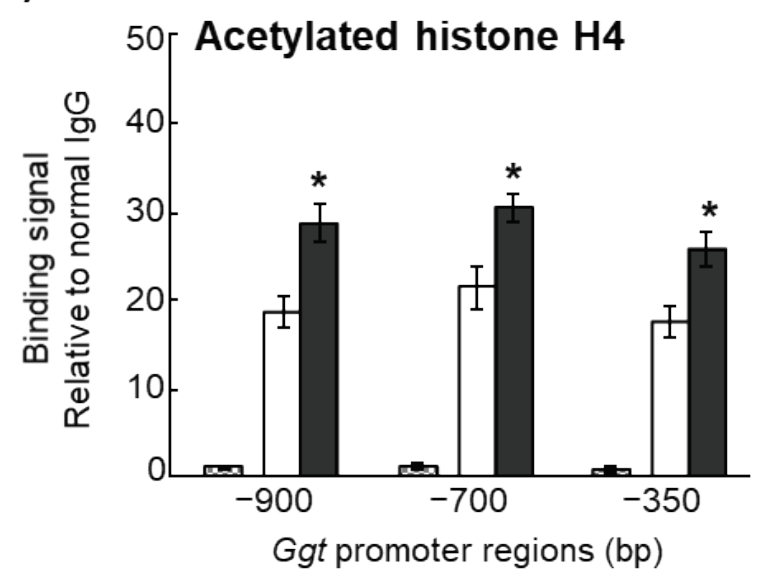

(F)

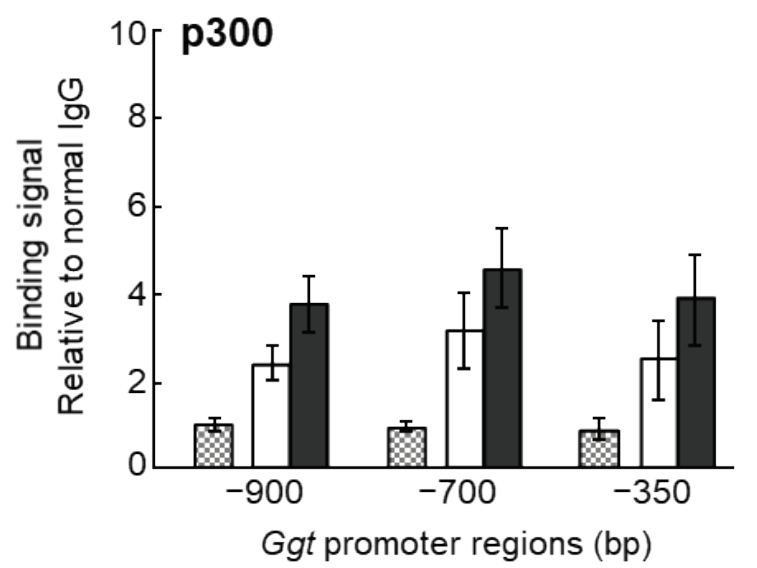

Normal lgG $\square$ Control $\square$ STZ

Fig. 1. Hepatic expression of Ggt/GGT, acetylation of histones $\mathrm{H} 3$ and $\mathrm{H} 4$, and bindings of CBP and p300 to the Ggt gene promoter in control and STZ-induced moderate hypoinsulinemic rats. (A) Expression levels of Pck1, Gpt, and Ggt genes were analyzed using realtime quantitative PCR. Expression levels were normalized to the expression of 36b4. (B) Expression level of GGT was detected using Immunoblot. The expression level was normalized to the expression of TBP. ChIP assays were performed using (C) anti-acetylated histone H3, (D) anti-acetylated histone H4, (E) anti-CBP, (F) anti-p300, or normal IgG. ChIP signals were detected using real-time qPCR with primers for the designated promoter regions of Ggt. Values are expressed as means \pm standard error of the mean ( $n=6-7)$. $* * * P<0.001 ; * * P<0.01 ; * P<0.05$, significantly different from the control group (Student's $t$-test). Control, control rats treated with vehicle; STZ, moderate hypoinsulinemic rats treated with streptozotocin. 
Furthermore, it is noteworthy that the moderate hypoinsulinemic rats exhibited higher serum glucagon levels in this study. It has been described that glucagon increases the recruitment of CBP to hepatic gluconeogenic genes under fasting conditions via activation of the cAMP/PKA/CREB signaling pathway (Altarejos and Montminy 2011). Considering these findings, it is speculated that a combination of lower insulin and higher glucagon could induce expression of the Ggt gene via enhanced recruitment of CBP to the Ggt gene; however, this hypothesis warrants further investigation.

Though the present study demonstrated the enhanced expression of hepatic Ggt/GGT in STZ-induced moderate hypoinsulinemic rats, the relationship between moderate hypoinsulinemia and GGT remains controversial. Studies in humans have reported that elevated GGT levels could predict the development of insulin resistance (Lee et al. 2013, Ryoo et al. 2014).
Thus, further studies should chronologically investigate circulating levels of GGT accompanied by pancreatic $\beta$-cell exhaustion using young Goto-Kakizaki type 2 diabetic rats, which exhibit mild hyperglycemia with a reduction of $\beta$-cell mass at an early stage (Movassat et al. 1995).

In conclusion, we have demonstrated that enhanced hepatic Ggt expression is associated with increased CBP binding and histone $\mathrm{H} 3$ and $\mathrm{H} 4$ acetylation on the Ggt gene promoter regions in STZ-induced moderate hypoinsulinemic rats.

\section{Conflict of Interest}

There is no conflict of interest.

\section{Acknowledgements}

This work was supported by JSPS KAKENHI Grant number JP18K02237.

\section{References}

ALTAREJOS JY, MONTMINY M: CREB and the CRTC co-activators: Sensors for hormonal and metabolic signals. Nat Rev Mol Cell Biol 12: 141-151, 2011. https://doi.org/10.1038/nrm3072

ARKKILA PE, KOSKINEN PJ, KANTOLA IM, RÖNNEMAA T, SEPPÄNEN E, VIIKARI JS: Diabetic complications are associated with liver enzyme activities in people with type 1 diabetes. Diabetes Res Clin Pract 52: 113-118, 2001. https://doi.org/10.1016/S0168-8227(00)00241-2

CHAN HM, LA THANGUE NB: p300/CBP proteins: HATs for transcriptional bridges and scaffolds. J Cell Sci 114 : 2363-2373, 2001.

KUNUTSOR SK, ABBASI A, ADLER AI: Gamma-glutamyl transferase and risk of type II diabetes: an updated systematic review and dose-response meta-analysis. Ann Epidemiol 24: 809-816, 2014. https://doi.org/10.1016/j.annepidem.2014.09.001

KUNUTSOR SK: Gamma-glutamyltransferase-friend or foe within? Liver Int 36: 1723-1734, 2016. https://doi.org/10.1111/liv.13221

HE L, SABET A, DJEDJOS S, MILLER R, SUN X, HUSSAIN MA, RADOVICK S, WONDISFORD FE: Metformin and insulin suppress hepatic gluconeogenesis through phosphorylation of CREB binding protein. Cell 137: 635-646, 2009. https://doi.org/10.1016/i.cell.2009.03.016

HONMA K, KAMIKUBO M, MOCHIZUKI K, GODA T: Insulin-induced inhibition of gluconeogenesis genes, including glutamic pyruvic transaminase 2, is associated with reduced histone acetylation in a human liver cell line. Metabolism 71: 118-124, 2017. https://doi.org/10.1016/j.metabol.2017.03.009

ICHIGO Y, TAKESHITA A, HIBINO M, NAKAGAWA T, HAYAKAWA T, PATEL D, FIELD CJ, SHIMADA M: High-fructose diet-induced hypertriglyceridemia is associated with enhanced hepatic expression of ACAT2 in rats. Physiol Res 68: 1021-1026, 2019. https://doi.org/10.33549/physiolres.934226

LEE SY, SUNG E, CHANG Y: Elevated serum gamma-glutamyltransferase is a strong marker of insulin resistance in obese children. Int J Endocrinol 2013: 578693, 2013. https://doi.org/10.1155/2013/578693

MOVASSAT J, SAULNIER C, PORTHA B: Beta-cell mass depletion precedes the onset of hyperglycaemia in the GK rat, a genetic model of non-insulin-dependent diabetes mellitus. Diabete Metab 21: 365-370, 1995. 
RAVNSKJAER K, HOGAN MF, LACKEY D, TORA L, DENT SYR, OLEFSKY J, MONTMINY M: Glucagon regulates gluconeogenesis through KAT2B- and WDR5-mediated epigenetic effects. J Clin Invest 123: 4318-4328, 2013. https://doi.org/10.1172/JCI69035

ROH TY, CUDDAPAH S, ZHAO K: Active chromatin domains are defined by acetylation islands revealed by genome-wide mapping. Genes Dev 19: 542-552, 2005. https://doi.org/10.1101/gad.1272505

RYOO JH, OH CM, KIM HS, PARK SK, CHOI JM: Clinical association between serum gamma-glutamyltransferase levels and the development of insulin resistance in Korean men: a 5-year follow-up study. Diabet Med 31: 455-461, 2014. https://doi.org/10.1111/dme.12315

SCHÜBELER D, MACALPINE DM, SCALZO D, WIRBELAUER C, KOOPERBERG C, VAN LEEUWEN F, GOTTSCHLING DE, O'NEILL LP, TURNER BM, DELROW J, BELL SP, GROUDINE M: The histone modification pattern of active genes revealed through genome-wide chromatin analysis of a higher eukaryote. Genes Dev 18: 1263-1271, 2004. https://doi.org/10.1101/gad.1198204

SHIMADA M, ICHIGO Y, SHIROUCHI B, TAKASHIMA S, INAGAKI M, NAKAGAWA T, HAYAKAWA T: Treatment with myo-inositol attenuates binding of the carbohydrate-responsive element-binding protein to the ChREBP- $\beta$ and FASN genes in rat nonalcoholic fatty liver induced by high-fructose diet. Nutr Res 64: 49-55, 2019. https://doi.org/10.1016/j.nutres.2019.01.002

SHOUKRY MI: Gamma glutamyl transferase in diabetic rats. The effect of insulin treatment. Acta Diabetol Lat 25: 299-302, 1988. https://doi.org/10.1007/BF02581128

SUZUKI T, MURAMATSU T, MORIOKA K, GODA T, MOCHIZUKI K: ChREBP binding and histone modifications modulate hepatic expression of the Fasn gene in a metabolic syndrome rat model. Nutrition 31: 877-883, 2015. https://doi.org/10.1016/i.nut.2015.01.005

WANG L, ZHANG J, WANG B, ZHANG Y, HONG J, ZHANG Y, WANG W, GU W: New evidence for an association between liver enzymes and pancreatic islet beta-cell dysfunction in young obese patients. Endocrine 44: 688-695, 2013. https://doi.org/10.1007/s12020-013-9937-7 